\title{
Nonadiabatic coupling in cold collisions of spin- polarized metastable hydrogen atoms
}

\section{Citation}

Forrey, Robert C., Alex Dalgarno, Yulian V. Vanne, Alejandro Saenz, and Piotr Froelich. 2007. "Nonadiabatic Coupling in Cold Collisions of Spin-Polarized Metastable Hydrogen Atoms." Physical Review A 76 (5). https://doi.org/10.1103/physreva.76.052709.

\section{Permanent link}

http://nrs.harvard.edu/urn-3:HUL.InstRepos:41417418

\section{Terms of Use}

This article was downloaded from Harvard University's DASH repository, and is made available under the terms and conditions applicable to Other Posted Material, as set forth at http:// nrs.harvard.edu/urn-3:HUL.InstRepos:dash.current.terms-of-use\#LAA

\section{Share Your Story}

The Harvard community has made this article openly available.

Please share how this access benefits you. Submit a story.

Accessibility 


\title{
Nonadiabatic coupling in cold collisions of spin-polarized metastable hydrogen atoms
}

\author{
Robert C. Forrey \\ Department of Physics, Pennsylvania State University, Berks Campus, Reading, Pennsylvania 19610-6009, USA \\ Alex Dalgarno \\ Harvard-Smithsonian Center for Astrophysics, 60 Garden Street, Cambridge, Massachusetts 02138, USA \\ Yulian V. Vanne and Alejandro Saenz \\ AG Moderne Optik, Institut für Physik, Humboldt-Universität zu Berlin, Hausvogteiplatz 5-7, D-10117 Berlin, Germany
}

Piotr Froelich

Department of Quantum Chemistry, Uppsala University, Box 518, 75120 Uppsala, Sweden

(Received 31 July 2007; published 29 November 2007)

\begin{abstract}
Previous calculations of low-temperature cross sections for collisions between spin-polarized metastable hydrogen atoms are improved to include nonadiabatic radial and angular coupling at large interatomic separations. The electrostatic dipole-quadrupole interaction produces nonadiabatic radial coupling between $(2 s, 2 p)$ and $(2 p, 2 p)$ states, while the Coriolis interaction produces nonadiabatic angular coupling. Both of these long-range contributions are handled in a space-fixed atomic gauge that is particularly convenient for a spinpolarized system. The improved theoretical results are compared with an existing experiment.
\end{abstract}

DOI: 10.1103/PhysRevA.76.052709

\section{INTRODUCTION}

An important prerequisite for achieving the goal of highresolution spectroscopy of metastable hydrogen is a detailed understanding of the atomic collisions. Theoretical $[1,2]$ and experimental [3] studies of cold collisions between two spinpolarized $\mathrm{H}(2 s)$ atoms have been performed. Calculated trap loss rate coefficients are about 2 or 3 times larger than the experimental error bars and show little temperature variation in a region where the experiment suggests that there may be a significant decrease. The trap loss rate coefficient is a measure of the total collisional quenching efficiency and may contain contributions from single and double excitation transfer reactions and also associative and Penning ionization reactions:

$$
\begin{gathered}
\mathrm{H}(2 s)+\mathrm{H}(2 s) \rightarrow \mathrm{H}(2 s)+\mathrm{H}(2 p), \\
\mathrm{H}(2 s)+\mathrm{H}(2 s) \rightarrow \mathrm{H}(2 p)+\mathrm{H}(2 p), \\
\mathrm{H}(2 s)+\mathrm{H}(2 s) \rightarrow \mathrm{H}_{2}^{+}+e, \\
\mathrm{H}(2 s)+\mathrm{H}(2 s) \rightarrow \mathrm{H}+\mathrm{H}^{+}+e .
\end{gathered}
$$

A recent investigation [4] of doubly excited autoionizing states of $\mathrm{H}_{2}$ converging to the $\mathrm{H}(n=2)+\mathrm{H}\left(n^{\prime}=2\right)$ limit revealed that significant nonadiabatic radial coupling occurs at large internuclear separation $R$. This coupling originates from a dipole-quadrupole interaction that yields nonzero, $R$-dependent matrix elements between the $(2 s, 2 p)$ and $(2 p, 2 p)$ states. The diabatic scattering formulation that has been applied to this system [1,2] relied on a unitary transformation between atomic and molecular basis states that is independent of $R$. Although this transformation is valid at large internuclear separations, it cannot accurately account for the molecular behavior at small $R$. Therefore, coupling to the $(2 s, 2 p)$ state, which vanishes at leading order in an ex-
PACS number(s): 34.50.Ez

pansion in inverse powers of $R$, was artificially assumed to be zero for all $R$. In order to extend the diabatic formulation to include the $(2 s, 2 p)$ coupling, it is necessary to allow a radial coordinate dependence in the transformation between atomic and molecular basis states. The required transformation has been given previously and a modified diabatic basis set was suggested [4]. In the present work, we employ the modified basis set and the $R$-dependent transformation to see whether the inelastic scattering cross sections may be brought into better agreement with experiment [3].

The scattering formulation used in our previous calculations $[1,2]$ is another source of possible error that is investigated in the present work. The centrifugal sudden or coupled-states (CS) approximation [5] that was used assumes that the orbital angular momentum of the nuclear motion is decoupled from the internal angular momentum of the atoms. This decoupling of angular momenta is equivalent to performing the scattering calculation in the body-fixed frame while neglecting the Coriolis interaction. It is also equivalent to performing the scattering calculation in a space-fixed frame while neglecting nonadiabatic angular couplings that persist at large distances [6]. Improving the CS formulation requires either (i) including the Coriolis coupling in a scattering calculation in the body-fixed frame and then transforming to the space-fixed frame to match to the appropriate boundary condition or (ii) making a gauge transformation of the scattering equations in a space-fixed frame that removes all nonadiabatic angular couplings that may occur in the separated atom limit [6]. Method (ii) has the advantage that the polarization direction may be defined relative to a spacefixed axis. This is particularly convenient for spin-polarized systems and is the method followed here. This paper is organized as follows. In Sec. II, a general scattering formulation is described that allows either method (i) or (ii) to be applied along with the molecular to atomic gauge transformation that is needed to implement method (ii). Sections III and IV give the atomic basis sets and adiabatic potentials that 
are used in this work. Sections V and VI describe how the nonadiabatic radial and angular couplings are included in the calculations. Sections VII and VIII provide numerical results and discussion. Comparisons are made with previous calculations and with an existing experiment.

\section{SCATTERING FORMULATION}

In order to describe the approach that will be taken to include nonadiabatic coupling, it is necessary to review the scattering formulation. Let $\vec{r}_{1}$ and $\vec{r}_{2}$ be the position vectors of the two electrons with respect to the center of mass of the nuclei and $R_{\infty}$ be an internuclear separation that is large enough that the system is well approximated as two isolated atoms. The scattering calculations may be performed in the diabatic representation [7], which uses a coupled-channels expansion of the form

$$
\Psi\left(\vec{r}_{1}, \vec{r}_{2} ; \vec{R}\right)=\sum_{n} \phi_{n}\left(\vec{r}_{1}, \vec{r}_{2} ; \vec{R}_{\infty}\right) u_{n}(\vec{R}),
$$

where $\left\{\phi_{n}\right\}$ is an orthonormal basis set of products of atomic functions. This basis set is described in Sec. III. The Schrödinger equation for the nuclear motion is

$$
\begin{gathered}
\left(-\frac{1}{2 \mu} \nabla_{R}^{2}-E\right) u_{n}(\vec{R})=-\sum_{m} V_{n m}(\vec{R}) u_{m}(\vec{R}), \\
V_{n m}(\vec{R})=\left\langle\phi_{n}\left(\vec{r}_{1}, \vec{r}_{2} ; \vec{R}_{\infty}\right)\left|H_{e l}\left(\vec{r}_{1}, \vec{r}_{2} ; \vec{R}\right)\right| \phi_{m}\left(\vec{r}_{1}, \vec{r}_{2} ; \vec{R}_{\infty}\right)\right\rangle .
\end{gathered}
$$

In the adiabatic representation, the electronic Hamiltonian is diagonalized for each $\vec{R}$ with molecular eigenfunctions $\xi_{n}\left(\vec{r}_{1}, \vec{r}_{2} ; \vec{R}\right)$ and eigenvalues $V_{n}(\vec{R})$ :

$$
H_{e l}\left(\vec{r}_{1}, \vec{r}_{2} ; \vec{R}\right)=\sum_{i}\left|\xi_{i}\left(\vec{r}_{1}, \vec{r}_{2} ; \vec{R}\right)\right\rangle V_{i}(\vec{R})\left\langle\xi_{i}\left(\vec{r}_{1}, \vec{r}_{2} ; \vec{R}\right)\right| .
$$

Substituting (8) into (7) yields

$$
\begin{aligned}
V_{n m}(\vec{R})= & \sum_{i}\left\langle\phi_{n}\left(\vec{r}_{1}, \vec{r}_{2} ; \vec{R}_{\infty}\right) \mid \xi_{i}\left(\vec{r}_{1}, \vec{r}_{2} ; \vec{R}\right)\right\rangle V_{i}(\vec{R}) \\
& \times\left\langle\xi_{i}\left(\vec{r}_{1}, \vec{r}_{2} ; \vec{R}\right) \mid \phi_{m}\left(\vec{r}_{1}, \vec{r}_{2} ; \vec{R}_{\infty}\right)\right\rangle .
\end{aligned}
$$

Small energy corrections such as the fine structure and/or Lamb shifts may be conveniently included at long range by transforming the atomic basis $\phi_{n}$ to the total angular momentum representation $\psi_{n}$ to yield

$$
\begin{aligned}
V_{n m}^{\prime}(\vec{R})= & \sum_{i j k}\left\langle\psi_{n}\left(\vec{r}_{1}, \vec{r}_{2} ; \vec{R}_{\infty}\right) \mid \phi_{i}\left(\vec{r}_{1}, \vec{r}_{2} ; \vec{R}_{\infty}\right)\right\rangle \\
& \times\left\langle\phi_{i}\left(\vec{r}_{1}, \vec{r}_{2} ; \vec{R}_{\infty}\right) \mid \xi_{j}\left(\vec{r}_{1}, \vec{r}_{2} ; \vec{R}\right)\right\rangle V_{j}(\vec{R}) \\
& \times\left\langle\xi_{j}\left(\vec{r}_{1}, \vec{r}_{2} ; \vec{R}\right) \mid \phi_{k}\left(\vec{r}_{1}, \vec{r}_{2} ; \vec{R}_{\infty}\right)\right\rangle \\
& \times\left\langle\phi_{k}\left(\vec{r}_{1}, \vec{r}_{2} ; \vec{R}_{\infty}\right) \mid \psi_{m}\left(\vec{r}_{1}, \vec{r}_{2} ; \vec{R}_{\infty}\right)\right\rangle .
\end{aligned}
$$

The above formulation does not specify whether the coordinate system is body fixed or space fixed. If it is assumed to be body fixed with the $z$ axis along the direction of $\hat{R}$, then the relative nuclear orbital angular momentum $\vec{l}$ will be per- pendicular to the $z$ axis and the potential matrix elements (9) and (10) will be independent of $l$ and the projection quantum number $m_{l}$ will be zero. Because $\hat{l}^{2}$ operates on the angular part of the body-fixed wave function, the usual centrifugal potential becomes the Coriolis potential [5]

$$
V_{C}=\frac{1}{2 \mu R^{2}}\left[\hat{J}^{2}+\hat{j}^{2}-2 \hat{J}_{z} \hat{j}_{z}-\hat{J}_{+} \hat{j}_{-}-\hat{J}_{-} \hat{j}_{+}\right],
$$

where $\vec{J}=\vec{j}+\vec{l}$ is the total angular momentum for a system with total internal angular momentum $\vec{j}$ and where $\hat{J}_{ \pm}$and $\hat{j}_{ \pm}$ are the usual ladder operators. Matrix elements of $V_{C}$ may be expressed in the total angular momentum $\psi$ representation as

$$
\begin{gathered}
V_{C}(\Omega, \Omega)=\frac{1}{2 \mu R^{2}}\left[J(J+1)+j(j+1)-2 \Omega^{2}\right], \\
V_{C}(\Omega, \Omega \pm 1) \\
=-\frac{1}{2 \mu R^{2}} \sqrt{J(J+1)-\Omega(\Omega \pm 1)} \sqrt{j(j+1)-\Omega(\Omega \pm 1)},
\end{gathered}
$$

where $\Omega$ is the body-fixed projection of both $J$ and $j$. The CS approximation is made by neglecting the off-diagonal matrix elements (13) and assuming the nuclear orbital angular momentum is conserved with all of the centrifugal barriers that may occur for a given $J$ in Eq. (12) replaced by a single barrier [5]. These approximations may be systematically removed by adding the exact terms (12) and (13) to the potential coupling (10) and solving the resulting coupled-channels Schrödinger equation.

An alternative approach [6] is to assume that $\vec{R}$ is aligned along the space-fixed $z$ axis. The electronic Hamiltonian (8) may be written

$$
\begin{aligned}
H_{e l}\left(\vec{r}_{1}, \vec{r}_{2} ; \vec{R}\right)= & T_{M}(\hat{R}) H_{e l}\left(\vec{r}_{1}, \vec{r}_{2} ; R\right) T_{M}^{-1}(\hat{R})=\sum_{i} T_{M}(\hat{R}) \\
& \times\left|\xi_{i}\left(\vec{r}_{1}, \vec{r}_{2} ; R\right)\right\rangle V_{i}(R)\left\langle\xi_{i}\left(\vec{r}_{1}, \vec{r}_{2} ; R\right)\right| T_{M}^{-1}(\hat{R}),
\end{aligned}
$$

where $T_{M}(\hat{R})$ is a single-centered rotation operator that generates electron rotations about the nuclear center of mass. The diabatic scattering formulation will remain valid if it includes a gauge transformation $u_{n}(\vec{R}) \rightarrow T_{A}(\hat{R}) u_{n}(\vec{R})$, which removes nonadiabatic angular couplings that persist at large $R$ in the molecular gauge [6]. The operator $T_{A}(\hat{R})$ is a twocentered rotation operator that generates electron rotations about the atomic centers. The gauge transformation is an $\vec{R}$-dependent unitary transformation that redefines the basis states

$$
\begin{aligned}
\phi_{n}\left(\vec{r}_{1}, \vec{r}_{2} ; \vec{R}_{\infty}\right) & =T_{M}(\hat{R}) \phi_{n}\left(\vec{r}_{1}, \vec{r}_{2} ; R_{\infty}\right) \\
& \rightarrow T_{M}(\hat{R}) \phi_{n}\left(\vec{r}_{1}, \vec{r}_{2} ; R_{\infty}\right) T_{A}^{-1}(\hat{R})
\end{aligned}
$$

and replaces the potential matrix (9) by 


$$
\begin{aligned}
V_{n m}(\vec{R})= & \sum_{i} T_{A}(\hat{R})\left\langle\phi_{n}\left(\vec{r}_{1}, \vec{r}_{2} ; R_{\infty}\right) \mid \xi_{i}\left(\vec{r}_{1}, \vec{r}_{2} ; R\right)\right\rangle V_{i}(R) \\
& \times\left\langle\xi_{i}\left(\vec{r}_{1}, \vec{r}_{2} ; R\right) \mid \phi_{m}\left(\vec{r}_{1}, \vec{r}_{2} ; R_{\infty}\right)\right\rangle T_{A}^{-1}(\hat{R}),
\end{aligned}
$$

where the basis functions $\xi$ and $\phi$ are now independent of $\hat{R}$. The action of $T_{A}^{-1}(\hat{R})$ on the atomic basis states induces a rotation about the atomic centers that exactly compensates for the rotation induced by $T_{M}(\hat{R})$, allowing the basis states to be quantized along the space-fixed $z$ axis for arbitrary impact parameter [6]. The CS approximation is arrived at in the space-fixed frame by neglecting this angular coordinate dependence and setting the rotation operator to unity. This is equivalent to performing the scattering calculation in the molecular gauge and neglecting the long-range nonadiabatic angular coupling. The unitary matrix element

$$
U_{n m}(R) \equiv\left\langle\phi_{n}\left(\vec{r}_{1}, \vec{r}_{2} ; R_{\infty}\right) \mid \xi_{m}\left(\vec{r}_{1}, \vec{r}_{2} ; R\right)\right\rangle
$$

is a projection coefficient computed in [4]. The radial coordinate dependence contained in this matrix element is needed to include the nonadiabatic radial coupling between the $(2 s, 2 p)$ and $(2 p, 2 p)$ states. Going beyond the CS approximation and also including the nonadiabatic radial coupling, therefore, requires a full account of the nuclear coordinate dependence of each term in Eq. (16).

For cold collisions, the atomic fine structure and Lamb shift splittings are important and it is more convenient to perform the scattering calculation in the $\psi$ representation where the atomic spin-orbit interaction is diagonal. In this representation, the rotation operator $T(\hat{R})$ may be written in terms of a Wigner $D$ matrix [6] so that Eq. (10) is replaced by

$$
\begin{aligned}
V_{n m}^{\prime}(\vec{R})= & \sum_{i j k} \sum_{\Omega} D_{\Omega_{n}, \Omega}^{j_{n}}(\phi, \theta,-\phi) D_{\Omega, \Omega_{m}}^{j_{m}}(\phi,-\theta,-\phi) V_{j}(R) \\
& \times\left\langle\psi_{n}\left(\vec{r}_{1}, \vec{r}_{2} ; R_{\infty}\right) \mid \phi_{i}\left(\vec{r}_{1}, \vec{r}_{2} ; R_{\infty}\right)\right\rangle \\
& \times\left\langle\phi_{i}\left(\vec{r}_{1}, \vec{r}_{2} ; R_{\infty}\right) \mid \xi_{j}\left(\vec{r}_{1}, \vec{r}_{2} ; R\right)\right\rangle \\
& \times\left\langle\xi_{j}\left(\vec{r}_{1}, \vec{r}_{2} ; R\right) \mid \phi_{k}\left(\vec{r}_{1}, \vec{r}_{2} ; R_{\infty}\right)\right\rangle \\
& \times\left\langle\phi_{k}\left(\vec{r}_{1}, \vec{r}_{2} ; R_{\infty}\right) \mid \psi_{m}\left(\vec{r}_{1}, \vec{r}_{2} ; R_{\infty}\right)\right\rangle,
\end{aligned}
$$

upon application of the gauge transformation. In Eq. (18), $j_{n}$ is the total internal angular momenta of state $n$, whereas the indices $i, j, k, m$, and $n$ each refer to a state characterized by a set of quantum numbers. This notation becomes rather clumsy, so in the remaining sections the subscripts on the states will be dropped and the difference between states will be denoted by primes. The next section provides the details of each of the representations used in this work.

\section{ATOMIC BASIS SETS}

The atomic basis set in the $\phi$ representation described above may be defined by the quantum numbers $\left\{l_{a}, m_{a}, l_{b}, m_{b}, S, \Sigma\right\}$ where $S$ and $\Sigma$ are the total electronic spin and spin projection along the $z$ axis of the space-fixed coordinate system. The electronic orbital angular momentum and projection are denoted by $l_{a}, m_{a}$ and $l_{b}, m_{b}$ for atoms $a$ and $b$, respectively. The $\psi$ representation described above may be defined by the set of quantum numbers $\left\{l_{a}, l_{b}, j_{a}, j_{b}, j, \Omega\right\}$ where $\vec{j}_{a}=\vec{l}_{a}+\vec{s}_{a}, \vec{j}_{b}=\vec{l}_{b}+\vec{s}_{b}$, and $\vec{j}=\vec{j}_{a}+\vec{j}_{b}$ is the total internal angular momentum with projection $\Omega$ along the space-fixed $z$ axis. Basis sets that are symmetric with respect to inversion of the nuclei may be defined as

$$
\begin{aligned}
|\phi\rangle= & \frac{1}{\sqrt{2\left(1+\delta_{l_{a}, l_{b}} \delta_{m_{a}, m_{b}}\right)}}\left\{\left|l_{a}, m_{a}, l_{b}, m_{b}\right\rangle+\rho_{\phi}\left|l_{b}, m_{b}, l_{a}, m_{a}\right\rangle\right\} \\
& \times|S, \Sigma\rangle, \\
|\psi\rangle= & \frac{1}{\sqrt{2\left(1+\delta_{\left.l_{a}, l_{b} \delta_{j_{a}, j_{b}}\right)}\right.}}\left\{\left|l_{a}, l_{b}, j_{a}, j_{b}\right\rangle+\rho_{\psi}\left|l_{b}, l_{a}, j_{b}, j_{a}\right\rangle\right\}|j, \Omega\rangle,
\end{aligned}
$$

where $\rho_{\phi}=p \sigma(-1)^{l_{a}+l_{b}}$ and $\rho_{\psi}=p(-1)^{l_{a}+l_{b}+1}$ with $p=+1(-1)$ for gerade (ungerade) symmetry and $\sigma=+1(-1)$ for $S$ $=0$ (1). The atomic states (19) and (20) are also assumed to be symmetrized with respect to electron exchange when computing overlaps with molecular states. The use of atomic states that are symmetry adapted with respect to the inversion operation $\vec{R} \rightarrow-\vec{R}$ allows the scattering calculation to be partitioned into even and odd values of nuclear orbital angular momentum when proton exchange is taken into account. For spin-polarized hydrogen collisions, the two atoms approach each other in a ${ }^{3} \Sigma_{u}^{+}$molecular state of $\mathrm{H}_{2}$ with $\Omega$ $=\Sigma=1$. Therefore, $p=-1$ for all of the symmetry-adapted atomic states (19) and (20) needed to describe this system. The basis set (20) is particularly convenient for performing numerical calculations because the spin-orbit interaction is diagonal in this representation. Previous calculations restricted the basis set to only $\Omega=1$ states $[1,2]$ consistent with the neglect of the Coriolis interaction. This restriction is lifted in the present work as $\Omega$ is allowed to take on the values $0, \pm 1, \pm 2, \pm 3$. A total of 36 states are used in the diabatic expansion. Quantum numbers of these states are given in Table I.

A unitary transformation between the two atomic representations (19) and (20) may be achieved using the identities

$$
\begin{aligned}
\left|l_{a}, l_{b}, j_{a}, j_{b}, j, \Omega\right\rangle= & \sum_{L S} \sum_{\Lambda \Sigma}\left(\begin{array}{ccc}
L & j & S \\
\Lambda & -\Omega & \Sigma
\end{array}\right)\left\{\begin{array}{ccc}
l_{a} & s_{a} & j_{a} \\
l_{b} & s_{b} & j_{b} \\
L & S & j
\end{array}\right\} \\
& \times\left[j, j_{a}, j_{b}, L, S\right]^{1 / 2}(-1)^{j+\Omega}\left|l_{a}, l_{b}, L, \Lambda, S, \Sigma\right\rangle,
\end{aligned}
$$

$$
|L, \Lambda\rangle=(-1)^{l_{b}-l_{a}-\Lambda} \sum_{m_{a}, m_{b}} \sqrt{2 L+1}\left(\begin{array}{ccc}
l_{a} & l_{b} & L \\
m_{a} & m_{b} & -\Lambda
\end{array}\right)\left|m_{a}, m_{b}\right\rangle
$$

where $[a, b, \ldots, c] \equiv(2 a+1)(2 b+1) \cdots(2 c+1)$ and $(\cdots)$ and $\{\cdots\}$ denote $3 j$ and $9 j$ symbols, respectively. The total electronic orbital angular momentum and its projection along the $z$ axis are defined by $\vec{L}=\vec{l}_{a}+\vec{l}_{b}$ and $\Lambda=m_{a}+m_{b}$. The atomic basis sets used in this work are given in Table I and the 
TABLE I. Atomic basis sets.

\begin{tabular}{|c|c|c|c|c|c|c|c|c|c|c|c|c|c|c|}
\hline \multirow[b]{2}{*}{$n$} & \multicolumn{7}{|c|}{$\phi$ representation } & \multicolumn{7}{|c|}{$\psi$ representation } \\
\hline & $\rho_{\phi}$ & $l_{a}$ & $m_{a}$ & $l_{b}$ & $m_{b}$ & $S$ & $\Sigma$ & $\rho_{\psi}$ & $l_{a}$ & $l_{b}$ & $j_{a}$ & $j_{b}$ & $j$ & $\Omega$ \\
\hline 1 & 1 & 0 & 0 & 0 & 0 & 1 & 1 & 1 & 0 & 0 & $1 / 2$ & $1 / 2$ & 1 & 1 \\
\hline 2 & 1 & 1 & 0 & 1 & 0 & 1 & 1 & 1 & 1 & 1 & $1 / 2$ & $1 / 2$ & 1 & 1 \\
\hline 3 & -1 & 0 & 0 & 1 & 0 & 1 & 1 & -1 & 1 & 1 & $1 / 2$ & $3 / 2$ & 1 & 1 \\
\hline 4 & 1 & 1 & 1 & 1 & -1 & 1 & 1 & 1 & 1 & 1 & $3 / 2$ & $1 / 2$ & 1 & 1 \\
\hline 5 & 1 & 1 & 0 & 1 & 1 & 1 & 0 & 1 & 1 & 1 & $1 / 2$ & $3 / 2$ & 2 & 1 \\
\hline 6 & -1 & 1 & 0 & 1 & 1 & 0 & 0 & 1 & 1 & 1 & $3 / 2$ & $3 / 2$ & 3 & 1 \\
\hline 7 & 1 & 1 & 1 & 1 & 1 & 1 & -1 & -1 & 0 & 1 & $1 / 2$ & $1 / 2$ & 1 & 1 \\
\hline 8 & 1 & 0 & 0 & 1 & 1 & 0 & 0 & 1 & 0 & 1 & $1 / 2$ & $3 / 2$ & 1 & 1 \\
\hline 9 & -1 & 0 & 0 & 1 & 1 & 1 & 0 & -1 & 0 & 1 & $1 / 2$ & $3 / 2$ & 2 & 1 \\
\hline 10 & 1 & 1 & 1 & 1 & 1 & 1 & 0 & -1 & 1 & 0 & $3 / 2$ & $1 / 2$ & 2 & 2 \\
\hline 11 & 1 & 1 & 0 & 1 & 1 & 1 & 1 & 1 & 1 & 1 & $3 / 2$ & $1 / 2$ & 2 & 2 \\
\hline 12 & -1 & 0 & 0 & 1 & 1 & 1 & 1 & 1 & 1 & 1 & $3 / 2$ & $3 / 2$ & 3 & 2 \\
\hline 13 & 1 & 1 & 1 & 1 & 1 & 1 & 1 & 1 & 1 & 1 & $3 / 2$ & $3 / 2$ & 3 & 3 \\
\hline 14 & 1 & 0 & 0 & 0 & 0 & 1 & 0 & 1 & 0 & 0 & $1 / 2$ & $1 / 2$ & 1 & 0 \\
\hline 15 & 1 & 1 & 0 & 1 & 0 & 1 & 0 & 1 & 1 & 1 & $1 / 2$ & $1 / 2$ & 1 & 0 \\
\hline 16 & -1 & 0 & 0 & 1 & 0 & 1 & 0 & 1 & 1 & 1 & $3 / 2$ & $3 / 2$ & 1 & 0 \\
\hline 17 & 1 & 1 & 1 & 1 & -1 & 1 & 0 & 1 & 1 & 1 & $3 / 2$ & $3 / 2$ & 3 & 0 \\
\hline 18 & 1 & 1 & 0 & 1 & -1 & 1 & 1 & 1 & 0 & 1 & $1 / 2$ & $1 / 2$ & 0 & 0 \\
\hline 19 & 1 & 1 & 0 & 1 & 1 & 1 & -1 & -1 & 0 & 1 & $1 / 2$ & $1 / 2$ & 1 & 0 \\
\hline 20 & -1 & 0 & 0 & 1 & -1 & 1 & 1 & -1 & 1 & 1 & $1 / 2$ & $3 / 2$ & 1 & 0 \\
\hline 21 & -1 & 0 & 0 & 1 & 1 & 1 & -1 & 1 & 0 & 1 & $1 / 2$ & $3 / 2$ & 1 & 0 \\
\hline 22 & -1 & 1 & 1 & 1 & -1 & 0 & 0 & -1 & 0 & 1 & $1 / 2$ & $3 / 2$ & 2 & 0 \\
\hline 23 & 1 & 0 & 0 & 1 & 0 & 0 & 0 & 1 & 1 & 1 & $1 / 2$ & $3 / 2$ & 2 & 0 \\
\hline 24 & 1 & 0 & 0 & 0 & 0 & 1 & -1 & 1 & 0 & 0 & $1 / 2$ & $1 / 2$ & 1 & -1 \\
\hline 25 & 1 & 1 & 0 & 1 & 0 & 1 & -1 & 1 & 1 & 1 & $1 / 2$ & $1 / 2$ & 1 & -1 \\
\hline 26 & -1 & 0 & 0 & 1 & 0 & 1 & -1 & -1 & 1 & 1 & $1 / 2$ & $3 / 2$ & 1 & -1 \\
\hline 27 & 1 & 1 & 1 & 1 & -1 & 1 & -1 & 1 & 1 & 1 & $3 / 2$ & $3 / 2$ & 1 & -1 \\
\hline 28 & 1 & 1 & 0 & 1 & -1 & 1 & 0 & 1 & 1 & 1 & $1 / 2$ & $3 / 2$ & 2 & -1 \\
\hline 29 & -1 & 1 & 0 & 1 & -1 & 0 & 0 & 1 & 1 & 1 & $3 / 2$ & $3 / 2$ & 3 & -1 \\
\hline 30 & 1 & 1 & -1 & 1 & -1 & 1 & 1 & -1 & 0 & 1 & $1 / 2$ & $1 / 2$ & 1 & -1 \\
\hline 31 & 1 & 0 & 0 & 1 & -1 & 0 & 0 & 1 & 0 & 1 & $1 / 2$ & $3 / 2$ & 1 & -1 \\
\hline 32 & -1 & 0 & 0 & 1 & -1 & 1 & 0 & -1 & 0 & 1 & $1 / 2$ & $3 / 2$ & 2 & -1 \\
\hline 33 & 1 & 1 & -1 & 1 & -1 & 1 & 0 & -1 & 1 & 0 & $3 / 2$ & $1 / 2$ & 2 & -2 \\
\hline 34 & 1 & 1 & 0 & 1 & -1 & 1 & -1 & 1 & 1 & 1 & $3 / 2$ & $1 / 2$ & 2 & -2 \\
\hline 35 & -1 & 0 & 0 & 1 & -1 & 1 & -1 & 1 & 1 & 1 & $3 / 2$ & $3 / 2$ & 3 & -2 \\
\hline 36 & 1 & 1 & -1 & 1 & -1 & 1 & -1 & 1 & 1 & 1 & $3 / 2$ & $3 / 2$ & 3 & -3 \\
\hline
\end{tabular}

matrix elements of the $\phi \leftrightarrow \psi$ transformation in Table II. The symmetry of the atomic and molecular states with respect to reflection in the $x-z$ plane introduces an additional phase factor $(-1)^{L-\Lambda}$ that is important when applying the gauge transformation described in Sec. VI. This phase factor requires that the atomic states $\phi_{18}, \phi_{28}$, and $\phi_{34}$ be defined as the negative of $\phi_{11}, \phi_{5}$, and $\phi_{19}$, respectively.

\section{ADIABATIC POTENTIALS}

may be calculated using a multipole expansion of the form

$$
V_{e l}\left(\vec{r}_{1}, \vec{r}_{2} ; R\right)=\sum_{l, l^{\prime}} \sum_{\mu} A_{l, l^{\prime}}^{\mu} r_{1}^{l} r_{2}^{l^{\prime}} Y_{l, \mu}\left(\hat{r}_{1}\right) Y_{l^{\prime},-\mu}\left(\hat{r}_{2}\right) R^{-l-l^{\prime}-1}
$$

There are 64 atomic states corresponding to $n_{1}$ $=n_{2}=2$. The electrostatic coupling between these states where 


$$
A_{l, l^{\prime}}^{\mu}=\frac{4 \pi\left(l+l^{\prime}\right) !(-1)^{l^{\prime}}}{\left[(2 l+1)\left(2 l^{\prime}+1\right)(l-\mu) !(l+\mu) !\left(l^{\prime}-\mu\right) !\left(l^{\prime}+\mu\right) !\right]^{1 / 2}} .
$$

This allows the diabatic coupling matrix (7) to be diagonalized analytically and matched to $a b$ initio electronic structure calculations. Our electronic structure results provide a smooth match to the calculated long-range behavior [4]. Eleven adiabatic potential curves produce the $36 \times 36$ cou-

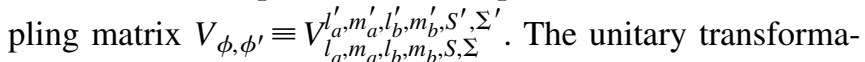
tion between the atomic $\phi$ and $\psi$ representations then yields the desired matrix $V_{\psi, \psi^{\prime}} \equiv V_{l_{a}, l_{b} l_{a}^{\prime}, l_{a}^{\prime}, j_{b}, j, \Omega}^{\prime}$. The long-range behavior of the 11 adiabatic potential curves is given below along with the corresponding symmetries. Comparison of the first nine potentials with previous calculations shows several improvements and corrections as described in [4]. The $O\left(R^{-7}\right)$ coefficients reported in [4] may also be included in the matching procedure if desired:

$$
\begin{gathered}
{ }^{3} \Sigma_{u}^{+}: V_{1}=-9 \sqrt{6} R^{-3}+324(8-3 \sqrt{6}) R^{-5}-6737 R^{-6}+O\left(R^{-7}\right), \\
{ }^{3} \Sigma_{u}^{+}: V_{2}=-6718 R^{-6}+O\left(R^{-7}\right), \\
{ }^{3} \Sigma_{u}^{+}: V_{3}=18 R^{-3}-3888 R^{-5}-8783 R^{-6}+O\left(R^{-7}\right), \\
{ }^{3} \Sigma_{u}^{+}: V_{4}=+9 \sqrt{6} R^{-3}+324(8+3 \sqrt{6}) R^{-5}-6737 R^{-6}+O\left(R^{-7}\right),
\end{gathered}
$$

$$
\begin{gathered}
{ }^{3} \Pi_{u}: V_{5}=432 R^{-5}-3062 R^{-6}+O\left(R^{-7}\right), \\
{ }^{1} \Pi_{u}: V_{6}=-7501 R^{-6}+O\left(R^{-7}\right), \\
{ }^{3} \Delta_{u}: V_{7}=216 R^{-5}-4042 R^{-6}+O\left(R^{-7}\right), \\
{ }^{3} \Pi_{u}: V_{8}=-9 R^{-3}-1296 R^{-5}-6165 R^{-6}+O\left(R^{-7}\right), \\
{ }^{1} \Pi_{u}: V_{9}=+9 R^{-3}-6165 R^{-6}+O\left(R^{-7}\right), \\
{ }^{1} \Sigma_{u}^{-}: V_{10}=-1824 R^{-6}+O\left(R^{-7}\right) \\
{ }^{1} \Sigma_{u}^{+}: V_{11}=-18 R^{-3}-8783 R^{-6}+O\left(R^{-7}\right) .
\end{gathered}
$$

The imaginary width components for the ${ }^{3} \Sigma_{u}^{+}$states are taken from Ref. [8], and those of the other symmetries are unknown and assumed to be zero. This introduces a source of possible error into the calculation of ionization cross sections. However, the neglected imaginary components couple only indirectly to the initial $(2 s, 2 s)$ state of interest, so we expect this error to be negligible.

\section{NONADIABATIC RADIAL COUPLING}

In previous work $[1,2]$, it was assumed that the unitary transformation matrix between atomic and molecular basis states was not dependent on distance-i.e.,

$$
U_{n m}(R) \approx\left\langle\phi_{n}\left(\vec{r}_{1}, \vec{r}_{2} ; R_{\infty}\right) \mid \xi_{m}\left(\vec{r}_{1}, \vec{r}_{2} ; R_{\infty}\right)\right\rangle .
$$

This approximation neglects nonadiabatic radial coupling between the $(2 s, 2 p)$ and $(2 p, 2 p)$ states. Using the full $R$ dependence in Eq. (17) allows the nonadiabatic radial coupling to be included within the diabatic formulation for an atomic basis set $\phi_{n}$ that is complete. The completeness of the atomic basis set may be measured as a function of distance by observing whether the numerical unitarity of the $U_{n m}(R)$ is maintained. Previous studies [4] showed that this measure of completeness for an atomic basis set consisting of $n_{1}=n_{2}$ $=2$ states is better than $99 \%$ for distances greater than 20 a.u. This represents a significant improvement over the use of approximation (36) where the neglected long-range coupling to the $(2 s, 2 p)$ state produced by the dipole-quadrupole interaction prevents an accurate treatment of the scattering at intermediate distances ( $\sim 20-200$ a.u.). In order to implement this improvement, it is necessary to match the eigenvector matrix elements obtained from the electronic structure calculations with those obtained from perturbation theory. This requires a total of 25 projection coefficients [4] in addition to the 11 potential curves (25)-(35).

The diabatic basis set is implicitly $R$ dependent, since it consists of atomic orbitals centered at the moving nuclei. This gives rise to weak radial derivative coupling which is small compared to the potential coupling. Figure 1 shows the potential coupling terms for ${ }^{3} \Pi_{u}$ symmetry with indices defined in Table I. Note that the matrix element $V_{5,9}$ between the $(2 p, 2 p)$ and $(2 s, 2 p)$ states was previously neglected $[1,2]$. This is the main effect of including the $R$ dependence in the projection coefficients. Similar results were found for the other symmetries. The computer code that performs the scattering calculation was written to allow an input value of $R$ where the $R$ dependence of the projection coefficients is first turned on. This introduces an adjustable parameter that may be used to study the sensitivity of the scattering problem to short distances where the atomic basis set is inadequate. The method described here for handling the radial coupling may also be implemented with the gauge transformation so that both nonadiabatic radial and angular couplings are included together. The details are given in the next section. 
TABLE II. Nonzero matrix elements of the $\phi \leftrightarrow \psi$ transformation.

\begin{tabular}{|c|c|c|c|c|c|c|c|c|}
\hline$i$ & $j$ & $\left\langle\phi_{j} \mid \psi_{i}\right\rangle$ & $i$ & $j$ & $\left\langle\phi_{j} \mid \psi_{i}\right\rangle$ & $j$ & $j$ & $\left\langle\phi_{j} \mid \psi_{i}\right\rangle$ \\
\hline 1 & 1 & 1 & 13 & 13 & 1 & 25 & 25 & $1 / 3$ \\
\hline 2 & 2 & $1 / 3$ & 14 & 14 & 1 & 25 & 28 & $-\sqrt{2 / 9}$ \\
\hline 2 & 5 & $-\sqrt{2 / 9}$ & 15 & 15 & $-1 / 3$ & 25 & 29 & $\sqrt{2 / 9}$ \\
\hline 2 & 6 & $-\sqrt{2 / 9}$ & 15 & 17 & $-\sqrt{2 / 9}$ & 25 & 30 & $2 / 3$ \\
\hline 2 & 7 & $2 / 3$ & 15 & 18 & $\sqrt{2 / 9}$ & 26 & 25 & $-1 / 3$ \\
\hline 3 & 2 & $-1 / 3$ & 15 & 19 & $\sqrt{2 / 9}$ & 26 & 27 & $\sqrt{1 / 2}$ \\
\hline 3 & 4 & $\sqrt{1 / 2}$ & 15 & 22 & $\sqrt{2 / 9}$ & 26 & 28 & $-\sqrt{1 / 18}$ \\
\hline 3 & 5 & $-\sqrt{1 / 18}$ & 16 & 15 & $-\sqrt{2 / 45}$ & 26 & 29 & $-\sqrt{2 / 9}$ \\
\hline 3 & 6 & $\sqrt{2 / 9}$ & 16 & 17 & $\sqrt{16 / 45}$ & 26 & 30 & $1 / 3$ \\
\hline 3 & 7 & $1 / 3$ & 16 & 18 & $-\sqrt{1 / 45}$ & 27 & 25 & $-\sqrt{8 / 45}$ \\
\hline 4 & 2 & $-\sqrt{8 / 45}$ & 16 & 19 & $-\sqrt{1 / 45}$ & 27 & 27 & $\sqrt{1 / 5}$ \\
\hline 4 & 4 & $\sqrt{1 / 5}$ & 16 & 22 & $\sqrt{5 / 9}$ & 27 & 28 & $\sqrt{1 / 45}$ \\
\hline 4 & 5 & $\sqrt{1 / 45}$ & 17 & 15 & $\sqrt{2 / 5}$ & 27 & 29 & $\sqrt{5 / 9}$ \\
\hline 4 & 6 & $-\sqrt{5 / 9}$ & 17 & 17 & $\sqrt{1 / 5}$ & 27 & 30 & $-\sqrt{2 / 45}$ \\
\hline 4 & 7 & $-\sqrt{2 / 45}$ & 17 & 18 & $\sqrt{1 / 5}$ & 28 & 25 & $\sqrt{1 / 3}$ \\
\hline 5 & 2 & $-\sqrt{1 / 3}$ & 17 & 19 & $\sqrt{1 / 5}$ & 28 & 27 & $\sqrt{1 / 6}$ \\
\hline 5 & 4 & $-\sqrt{1 / 6}$ & 18 & 16 & $\sqrt{1 / 3}$ & 28 & 28 & $-\sqrt{1 / 6}$ \\
\hline 5 & 5 & $\sqrt{1 / 6}$ & 18 & 20 & $-\sqrt{1 / 3}$ & 28 & 30 & $-\sqrt{1 / 3}$ \\
\hline 5 & 7 & $\sqrt{1 / 3}$ & 18 & 21 & $-\sqrt{1 / 3}$ & 29 & 25 & $\sqrt{4 / 15}$ \\
\hline 6 & 2 & $\sqrt{4 / 15}$ & 19 & 20 & $-\sqrt{1 / 3}$ & 29 & 27 & $\sqrt{2 / 15}$ \\
\hline 6 & 4 & $\sqrt{2 / 15}$ & 19 & 21 & $\sqrt{1 / 3}$ & 29 & 28 & $\sqrt{8 / 15}$ \\
\hline 6 & 5 & $\sqrt{8 / 15}$ & 19 & 23 & $\sqrt{1 / 3}$ & 29 & 30 & $\sqrt{1 / 15}$ \\
\hline 6 & 7 & $\sqrt{1 / 15}$ & 20 & 15 & $-2 / 3$ & 30 & 26 & $\sqrt{1 / 3}$ \\
\hline 7 & 3 & $-\sqrt{1 / 3}$ & 20 & 17 & $\sqrt{2 / 9}$ & 30 & 31 & $\sqrt{1 / 3}$ \\
\hline 7 & 8 & $\sqrt{1 / 3}$ & 20 & 18 & $\sqrt{1 / 18}$ & 30 & 32 & $-\sqrt{1 / 3}$ \\
\hline 7 & 9 & $\sqrt{1 / 3}$ & 20 & 19 & $\sqrt{1 / 18}$ & 31 & 26 & $-\sqrt{1 / 6}$ \\
\hline 8 & 3 & $\sqrt{1 / 6}$ & 20 & 22 & $-\sqrt{2 / 9}$ & 31 & 31 & $\sqrt{2 / 3}$ \\
\hline 8 & 8 & $\sqrt{2 / 3}$ & 21 & 20 & $\sqrt{1 / 6}$ & 31 & 32 & $\sqrt{1 / 6}$ \\
\hline 8 & 9 & $-\sqrt{1 / 6}$ & 21 & 21 & $-\sqrt{1 / 6}$ & 32 & 26 & $\sqrt{1 / 2}$ \\
\hline 9 & 3 & $\sqrt{1 / 2}$ & 21 & 23 & $\sqrt{2 / 3}$ & 32 & 32 & $\sqrt{1 / 2}$ \\
\hline 9 & 9 & $\sqrt{1 / 2}$ & 22 & 16 & $\sqrt{2 / 3}$ & 33 & 35 & 1 \\
\hline 10 & 12 & 1 & 22 & 20 & $\sqrt{1 / 6}$ & 34 & 33 & $-\sqrt{2 / 3}$ \\
\hline 11 & 10 & $\sqrt{2 / 3}$ & 22 & 21 & $\sqrt{1 / 6}$ & 34 & 34 & $\sqrt{1 / 3}$ \\
\hline 11 & 11 & $-\sqrt{1 / 3}$ & 23 & 18 & $-\sqrt{1 / 2}$ & 35 & 33 & $\sqrt{1 / 3}$ \\
\hline 12 & 10 & $\sqrt{1 / 3}$ & 23 & 19 & $\sqrt{1 / 2}$ & 35 & 34 & $\sqrt{2 / 3}$ \\
\hline 12 & 11 & $\sqrt{2 / 3}$ & 24 & 24 & 1 & 36 & 36 & 1 \\
\hline
\end{tabular}

\section{NONADIABATIC ANGULAR COUPLING}

A derivation of the molecular to atomic gauge transformation is given in the paper by Zygelman et al. [6]. The basic idea is as follows. In the molecular gauge, the assumption that $\vec{R}$ is aligned along the space-fixed $z$ axis requires the application of a rotation operator to generate electron rotations about the nuclear center of mass. In passing to the atomic gauge, a two-centered rotation operator must be applied to the asymptotic basis states so that they are quantized along the space-fixed $z$ axis for arbitrary orientation of the nuclei. The result is a coupling matrix of the form (18). When the angular part of the translational functions is inte- grated with Eq. (18) the resulting radial potential matrix becomes

$$
\begin{aligned}
V_{\tilde{\psi}, \tilde{\psi}^{\prime}}^{(J M)}(R) & \equiv\left\langle l_{a}, l_{b}, j_{a}, j_{b}, j, l, J, M\left|H_{e l}\right| l_{a}^{\prime}, l_{b}^{\prime}, j_{a}^{\prime}, j_{b}^{\prime}, j^{\prime}, l^{\prime}, J, M\right\rangle \\
& =\left[l, l^{\prime}\right]^{1 / 2} \sum_{\Omega}\left(\begin{array}{ccc}
j & l & J \\
\Omega & 0 & -\Omega
\end{array}\right)\left(\begin{array}{ccc}
j^{\prime} & l^{\prime} & J \\
\Omega & 0 & -\Omega
\end{array}\right) V_{\psi, \psi^{\prime}}(R),
\end{aligned}
$$

where $\vec{J}=\vec{j}+\vec{l}$ and $M=\Omega+m$. Note that $\tilde{\psi}$ represents the set of quantum numbers $\left\{l_{a}, l_{b}, j_{a}, j_{b}, j, l\right\}$ when referring to quantities in the total angular momentum representation whereas $\psi$ 


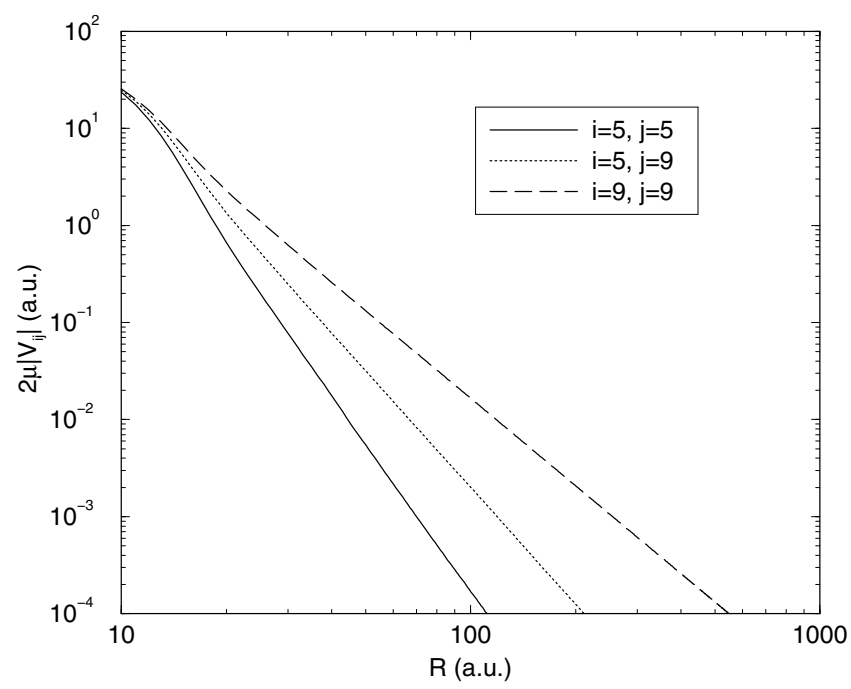

FIG. 1. Potential energy matrix elements as a function of distance for states with ${ }^{3} \Pi_{u}$ symmetry with indices defined in Table I. The off-diagonal matrix element, which couples the $(2 s, 2 p)$ and $(2 p, 2 p)$ states, is nonzero when the $R$ dependence of $U_{i j}$ is taken into account.

represents $\left\{l_{a}, l_{b}, j_{a}, j_{b}, j, \Omega\right\}$ as described in Sec. III.

An alternative approach [9] is to initially perform the gauge transformation using the $\phi$ representation to obtain the coupling matrix

$$
\begin{aligned}
V_{\tilde{\phi}, \widetilde{\phi}^{\prime}}^{(Q q)}(R) \equiv & \left\langle l_{a}, l_{b}, S, \Sigma, L, l, Q, q\left|H_{e l}\right| l_{a}^{\prime}, l_{b}^{\prime}, S^{\prime}, \Sigma^{\prime}, L^{\prime}, l^{\prime}, Q, q\right\rangle \\
= & {\left[l, l^{\prime}, L, L^{\prime}\right]^{1 / 2} \delta_{S, S^{\prime}} \delta_{\Sigma, \Sigma}, \sum_{\Lambda} \sum_{m_{a} m_{b}} \sum_{m_{a}^{\prime} m_{b}^{\prime}}\left(\begin{array}{ccc}
L & l & Q \\
\Lambda & 0 & -\Lambda
\end{array}\right) } \\
& \times\left(\begin{array}{ccc}
L^{\prime} & l^{\prime} & Q \\
\Lambda & 0 & -\Lambda
\end{array}\right)\left(\begin{array}{ccc}
l_{a} & l_{b} & L \\
m_{a} & m_{b} & -\Lambda
\end{array}\right) \\
& \times\left(\begin{array}{ccc}
l_{a}^{\prime} & l_{b}^{\prime} & L^{\prime} \\
m_{a}^{\prime} & m_{b}^{\prime} & -\Lambda
\end{array}\right) V_{\phi, \phi^{\prime}}(R),
\end{aligned}
$$

where $\vec{Q}=\vec{L}+\vec{l}$ with projection $q=\Lambda+m$. The desired $\psi$ representation may then be obtained using the transformation

$$
\begin{aligned}
V_{\tilde{\psi}, \tilde{\psi}^{\prime}}^{(J M)}(R)= & \sum_{Q q} \sum_{L \Lambda} \sum_{L^{\prime} \Lambda^{\prime}} \sum_{S \Sigma} \sum_{S^{\prime} \Sigma^{\prime}} \sum_{m \Omega} \sum_{m^{\prime} \Omega^{\prime}}[J, Q]\left(\begin{array}{ccc}
L & l & Q \\
\Lambda & m & -q
\end{array}\right) \\
& \times\left(\begin{array}{ccc}
L^{\prime} & l^{\prime} & Q \\
\Lambda^{\prime} & m^{\prime} & -q
\end{array}\right)\left\langle j_{a}, j_{b}, j, \Omega \mid S, \Sigma, L, \Lambda\right\rangle \\
& \times\left\langle S^{\prime}, \Sigma^{\prime}, L^{\prime}, \Lambda^{\prime} \mid j_{a}^{\prime}, j_{b}^{\prime}, j^{\prime}, \Omega^{\prime}\right\rangle\left(\begin{array}{ccc}
j & l & J \\
\Omega & m & -M
\end{array}\right) \\
& \times\left(\begin{array}{ccc}
j^{\prime} & l^{\prime} & J \\
\Omega^{\prime} & m^{\prime} & -M
\end{array}\right) V_{\tilde{\phi}, \widetilde{\phi}^{\prime}}^{(Q q)}(R) .
\end{aligned}
$$

Equations (37) and (39) were programmed separately and were found to give identical results. This potential contains coupling between the orbital angular momentum of the nuclei and the internal angular momentum of the atoms that is missing in the CS approximation. The nonadiabatic radial coupling described above may be included in (37) and (39) by allowing the $R$ dependence of $U_{n m}(R)$ to be computed prior to transforming to the $\psi$ representation.

The fine structure and Lamb shift contributions are included in the $\psi$ representation by adding the atomic energy defects $\Delta_{\psi}$ along the diagonal. The Schrödinger equation for the nuclear motion then reduces to the set of coupled radial equations

$$
\begin{aligned}
& -\frac{1}{2 \mu}\left[\frac{d^{2}}{d R^{2}}-\frac{l(l+1)}{R^{2}}\right] u_{\tilde{\psi}}^{J M}(R)+\sum_{\tilde{\psi}^{\prime}} V_{\tilde{\psi}, \tilde{\psi}^{\prime}}^{(J M)}(R) u_{\tilde{\psi}^{\prime}}^{J M}(R) \\
& \quad=\left(E-\Delta_{\tilde{\psi}}\right) u_{\tilde{\psi}}^{J M}(R) .
\end{aligned}
$$

The 36 atomic basis functions (see Table I) consist of 10 different combinations of $\left\{l_{a}, l_{b}, j_{a}, j_{b}, j\right\}$. The number of coupled channels needed for each $J$ may be computed from these 10 combinations and the $3 j$ symbols in Eq. (37). For $J=0,1$, and 2, there are 3,16, and 15 coupled channels, respectively. For $J>2$, there are 16 coupled channels when $J$ is even and 20 coupled channels when $J$ is odd. The next section describes results obtained by solving the coupled equations (40) and compares them with previous results [2] obtained using the CS approximation.

\section{COLLISION CROSS SECTIONS}

The collision cross sections computed within the CS approximation are obtained as in previous work [2] and are given by

$$
\begin{gathered}
\sigma_{\psi \rightarrow \psi^{\prime}}=\frac{2 \pi}{k_{\psi}^{2}} \sum_{l}(2 l+1)\left|T_{\psi \rightarrow \psi^{\prime}}^{(l)}\right|^{2}, \\
\sigma_{\psi \rightarrow \text { ionization }}=\frac{2 \pi}{k_{\psi}^{2}} \sum_{l}(2 l+1)\left[1-\sum_{\psi^{\prime}}\left|S_{\psi \rightarrow \psi^{\prime}}^{(l)}\right|^{2}\right] .
\end{gathered}
$$

Due to proton symmetrization, the summations in (41) and (42) are taken over even values of $l$ only. These cross sections are defined the same whether or not nonadiabatic radial coupling is included in the calculation. When the nonadiabatic angular coupling is included in the scattering formulation, however, we cannot simply sum over a conserved $l$ to obtain compact definitions for the cross sections. Instead, the cross sections may be derived using methods similar to those of Zygelman et al. [6]. The result is

$$
\begin{aligned}
& \sigma_{\psi \rightarrow \psi^{\prime}}=\frac{2 \pi}{k_{\psi}^{2}} \sum_{J M} \sum_{J^{\prime} M^{\prime}} \sum_{l m} \sum_{l^{\prime} m^{\prime}}\left(\begin{array}{ccc}
j & l & J \\
\Omega & m & -M
\end{array}\right)\left(\begin{array}{ccc}
j^{\prime} & l^{\prime} & J^{\prime} \\
\Omega^{\prime} & m^{\prime} & -M^{\prime}
\end{array}\right) \\
& \times\left(\begin{array}{ccc}
j & l & J^{\prime} \\
\Omega & m & -M^{\prime}
\end{array}\right)\left(\begin{array}{ccc}
j^{\prime} & l^{\prime} & J \\
\Omega^{\prime} & m^{\prime} & -M
\end{array}\right)\left[J, J^{\prime}\right]
\end{aligned}
$$

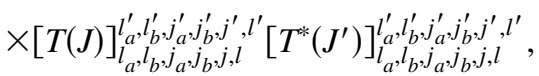




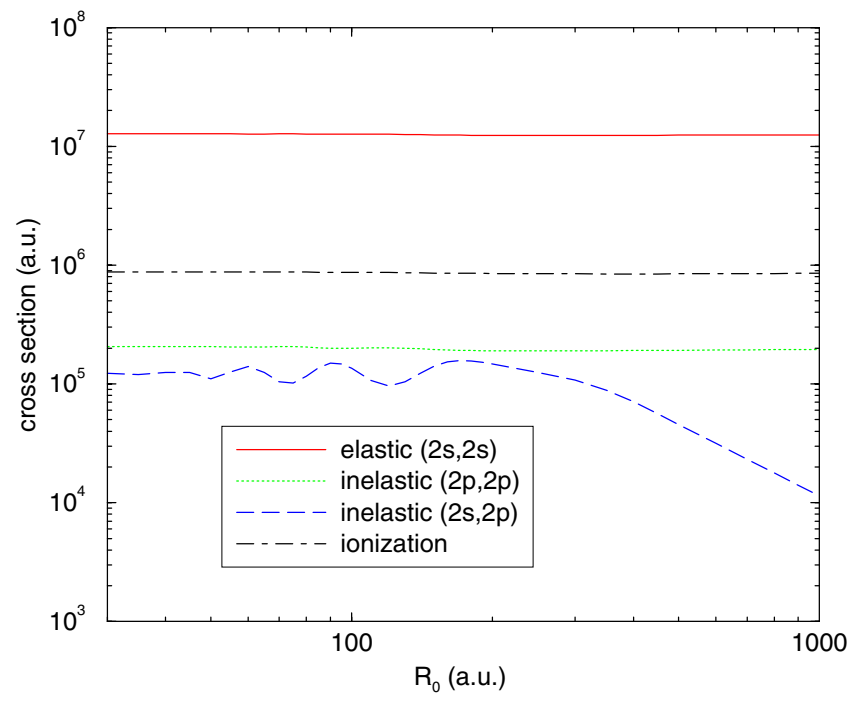

FIG. 2. (Color online) Cross sections computed with CS approximation at a collision energy of $10^{-10}$ a.u. The elastic, inelastic double excitation transfer, and ionization cross sections are not sensitive to the value of $R_{0}$ where the $R$ dependence of the transformation matrix $U_{i j}(R)$ is first turned on. In the $R_{0} \rightarrow \infty$ limit, the nonadiabatic radial coupling between the $(2 s, 2 p)$ and $(2 p, 2 p)$ states is completely neglected and the inelastic single excitation transfer cross section goes to zero. The inelastic and ionization cross sections in this limit are 2.5 times smaller and 1.2 times larger than their respective values obtained previously [2]. This difference is due to improvements in the adiabatic potentials (25)-(35) as described in [4].

$$
\begin{aligned}
\sigma_{\psi \rightarrow \text { ionization }}= & \frac{2 \pi}{k_{\psi}^{2}} \sum_{J M} \sum_{l l^{\prime} j^{\prime}}\left(\begin{array}{ccc}
j & l & J \\
\Omega & M-\Omega & -M
\end{array}\right)^{2}(2 J+1) \\
& \times\left\{1-\mid[S(J)]_{l_{a}, l_{b}, j_{a}, j_{b}, j, l}^{\left.l_{a}^{\prime}, l_{l}^{\prime}, j_{j}^{\prime}, j_{b}^{\prime}, j^{\prime},\left.l^{\prime}\right|^{2}\right\} .}\right.
\end{aligned}
$$

Proton symmetrization again restricts the summations in (43) and (44) over even values of $l$ and $l^{\prime}$ only. This differs from the formulation of Zygelman et al. [6] and is a consequence of using symmetry-adapted basis functions. Figure 2 shows cross sections computed within the CS approximation at a collision energy of $10^{-10}$ a.u. The horizontal axis is the distance $R_{0}$ where the $R$ dependence of the transformation matrix $U_{i j}(R)$ is first turned on. For $R<R_{0}$, the calculations assumed $U_{i j}(R)=U_{i j}\left(R_{0}\right)$. In the $R_{0} \rightarrow \infty$ limit, the nonadiabatic radial coupling is completely neglected and the cross sections may be compared to results obtained previously [2]. However, due to modifications in the long-range behavior of the adiabatic potentials (25)-(35), we find significant differences in this limit. The elastic, inelastic, and ionization cross sections are about 100 times larger, 2.5 times smaller, and 1.2 times larger than their respective values obtained using the previous long-range behavior which neglected coupling between $(2 s, 2 p)$ and $(2 p, 2 p)$ states. Large sensitivity of the elastic cross section to small changes in the potential is common in ultracold collisions. The new calculations also allow inelastic single excitation transfer to occur for finite values of $R_{0}$. Figure 2 shows that this contribution is relatively small

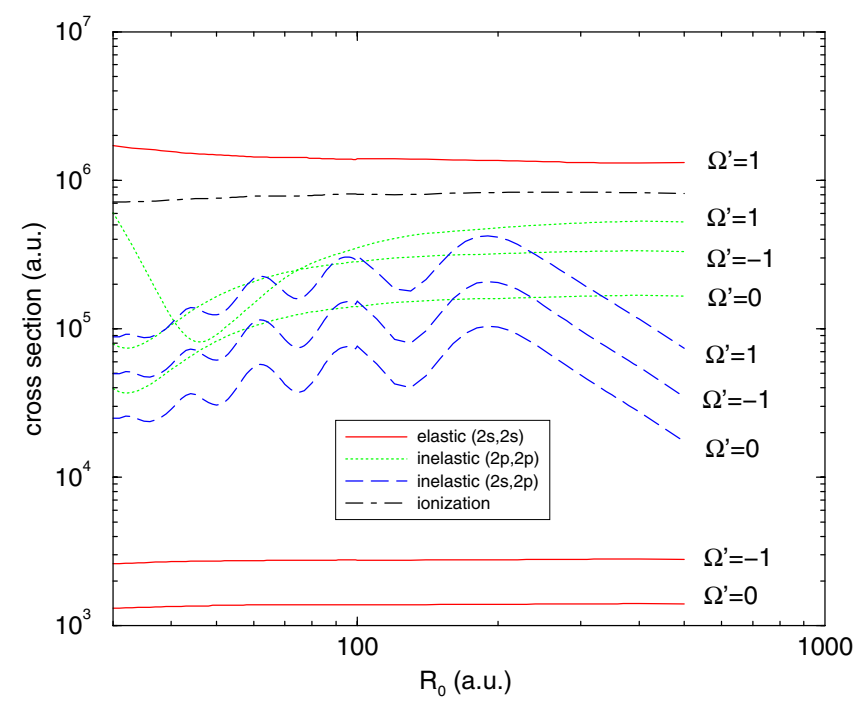

FIG. 3. (Color online) Cross sections computed in the atomic gauge at a collision energy of $10^{-10}$ a.u. The final-state projection quantum number $\Omega^{\prime}$ is not restricted to be the same as the initial value $\Omega$ as in the CS approximation. The single excitation transfer cross sections are relatively larger than in Fig. 2 with oscillations that are also larger in amplitude. The double excitation transfer cross section for $\Omega^{\prime}=1$ shows the strongest variation with $R_{0}$ at intermediate distances and has a minimum just below 50 a.u.

and contains oscillations that are presumably a measure of the sensitivity of the cross section to the short distance behavior. The low amplitude of these oscillations and the insensitivity of the remaining cross sections to $R_{0}$ suggest that nonadiabatic radial coupling does not play a significant role when the calculations are performed in the molecular gauge using the CS approximation.

The situation appears quite different when the cross sections are computed in the atomic gauge where uncalculated nonadiabatic angular terms have the proper long-range falloff. Figure 3 shows cross sections versus $R_{0}$ at the same collision energy as in Fig. 2. The curves are labeled by the final-state projection quantum number $\Omega^{\prime}$, which now may be different from the initial state $\Omega=1$. This yields three contributions that must be added together for each of the elastic and inelastic cross sections. More important is the observation that the $(2 s, 2 p)$ contributions are considerably larger than in Fig. 2 with oscillations that are of larger amplitudes. The inelastic $(2 p, 2 p)$ cross sections also show a significant $R_{0}$ dependence below 100 a.u. The sensitivity of the cross sections to $R_{0}$ suggests that the interaction at short distances is important at ultracold collision energies. When our calculations are extended to smaller values of $R_{0}$ the numerical unitarity begins to deteriorate and the corresponding cross sections become erratic. In this region, nonadiabatic angular coupling terms may be significant.

In the $R_{0} \rightarrow \infty$ limit, the inelastic and ionization cross sections are shifted from their respective CS values, but their sum is essentially unchanged. Therefore, it appears that the nonadiabatic angular coupling does not have a large effect on the total quenching cross section when the nonadiabatic radial coupling is neglected. It is only when the two sources of 


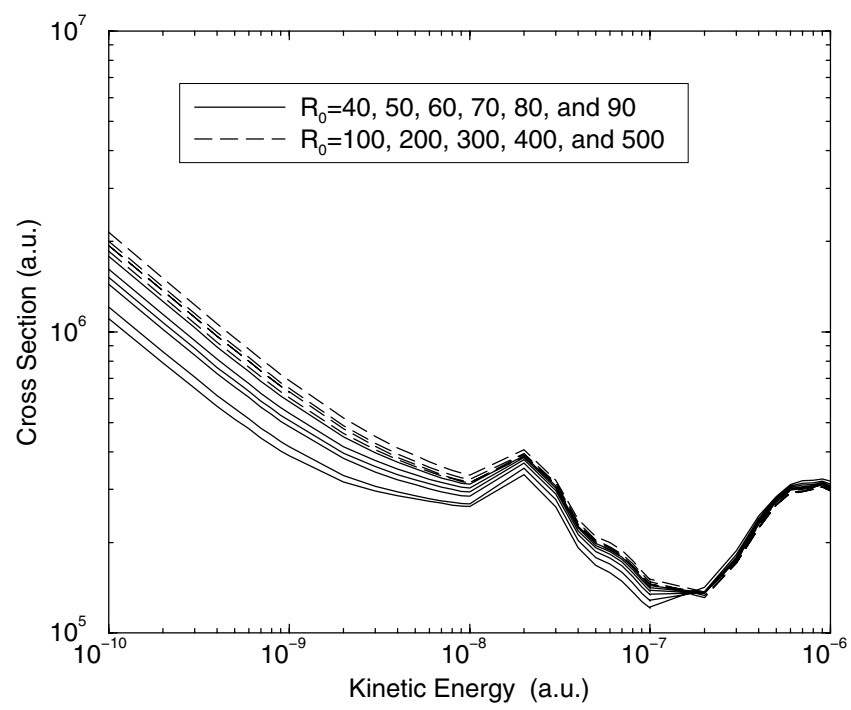

FIG. 4. Total quenching cross sections for $\mathrm{H}(2 s)+\mathrm{H}(2 s)$ collisions. The cross sections include both inelastic single and double excitation transfer contributions (including summation over all $\Omega^{\prime}$ ) and also ionization. The double excitation transfer and ionization cross sections have been multiplied by 2 to account for the loss of two metastable particles per collision. The solid curves are for starting distance $R_{0}=40-90$ a.u. and the dashed curves are for $R_{0}$ $=100-500$ a.u. as described in the text.

nonadiabatic coupling are taken together that a substantial change in the total cross section may appear. We may estimate the effect of the short-distance behavior by computing cross sections over a range of $R_{0}$. Figure 4 shows the total quenching cross section versus collision energy for $R_{0}$ $=40-500$ a.u. in steps of 10 a.u. for the low values (solid curves) and 100 a.u. for the high values (dashed curves). The total cross section includes both single and double excitation transfer contributions, including summation over $\Omega^{\prime}$, and also the ionization contribution. The double excitation transfer and ionization cross sections have been multiplied by 2 to account for the loss of two metastable particles per collision. The figure shows that there are only small differences in the curves when the energy is greater than $10^{-7}$ a.u. due to the long-range centrifugal barrier that prevents the atoms from getting into the region where the short-range coupling occurs. As the energy is decreased, the centrifugal barriers are reduced and the curves begin to separate from each other. The structure of each curve, however, remains very similar with a peak near $2 \times 10^{-8}$ a.u. that is due to a shape resonance. The shape resonance is a consequence of a very shallow well in the adiabatic potential curve for the $(2 s, 2 s)$ entrance channel produced by the long-range attraction. Similar resonances were found previously [2]; however, due to the use of the CS approximation, all centrifugal barriers that may occur for a given $J$ were replaced by a single barrier with angular momentum $l$. This typically causes an artificial enhancement or suppression of the shape resonance for a given partial wave. The approximation is removed by the molecular to atomic gauge transformation, and we see a significant difference in the cross sections in this energy region. The elastic scattering cross section (not shown) also shows sig-

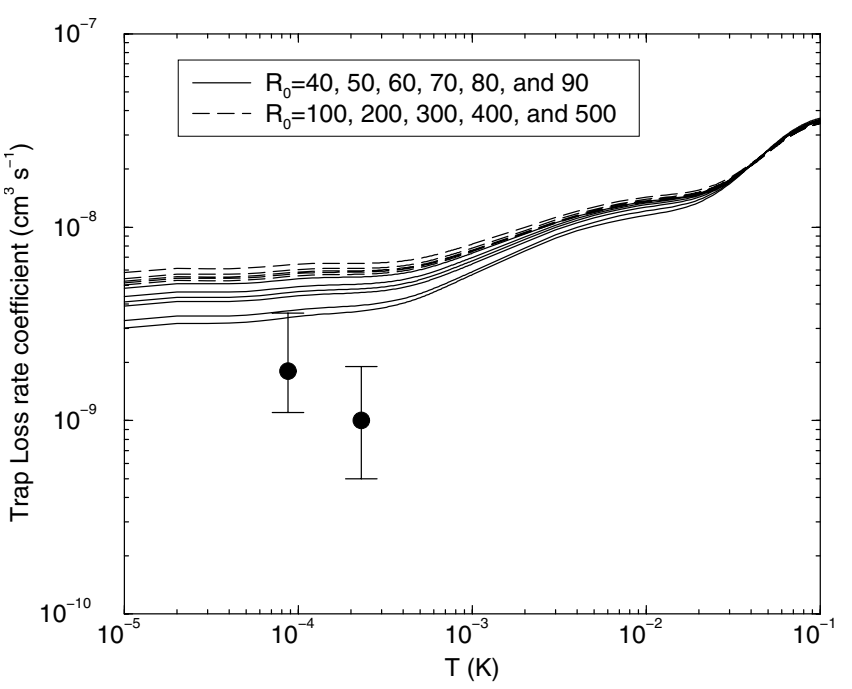

FIG. 5. Trap loss rate coefficients for $\mathrm{H}(2 s)+\mathrm{H}(2 s)$ collisions. The curves are computed by thermally averaging the product of total quenching cross section and collision velocity. The curves are labeled in the same way as Fig. 4. The points with error bars are experimental data taken from [3].

nificant differences from previous results [2] due to the improved handling of nonadiabatic angular coupling. At energies below the region of the shape resonance, the sensitivity of the cross sections to $R_{0}$ increases further. The dashed curves are larger than the solid curves in this energy region. The two lowest solid curves correspond to $R_{0}=40$ and 50 a.u., which is a consequence of the location of the minimum of the $\Omega^{\prime}=1$ curve for the inelastic $(2 p, 2 p)$ case shown in Fig. 3.

The cross sections shown in Fig. 4 may be used to compute trap loss rate coefficients by thermally averaging the product of cross section and collision velocity. The result is shown in Fig. 5 together with the experimental data points [3]. It is surprising that none of the theoretical curves are able to reconcile the disagreement with experiment. The decrease in the rate coefficients for $R_{0}<100$ a.u. suggests that neglected short-range nonadiabatic coupling may be contributing to the disagreement with experiment. The $R_{0}=40$ and 50 curves just barely intersect the upper part of the error bar for $T=100 \mu \mathrm{K}$, and all of the curves lie above the error bar for $T=200 \mu \mathrm{K}$. The shape resonance that was found in the cross section at an energy near $2 \times 10^{-8}$ a.u. contributes to an increasing rate coefficient for $T>1 \mathrm{mK}$. The long-range origin of this resonance makes it unlikely that it would disappear with an improved treatment of the short-range nonadiabatic coupling. The cross sections increase inversely with decreasing collision velocity for energies below $10^{-9}$ a.u. in accordance with Wigner's threshold law. This produces a trap loss rate coefficient that is nearly independent of temperature for $T<300 \mu \mathrm{K}$. Although the error bars of the two experimental data points allow for a rate coefficient that is independent of temperature in this region, the data suggest that there may be a decrease in the rate coefficient for $T$ $>100 \mu \mathrm{K}$. Figure 6 shows the energy dependence of the function that multiplies the cross section to obtain the ther- 


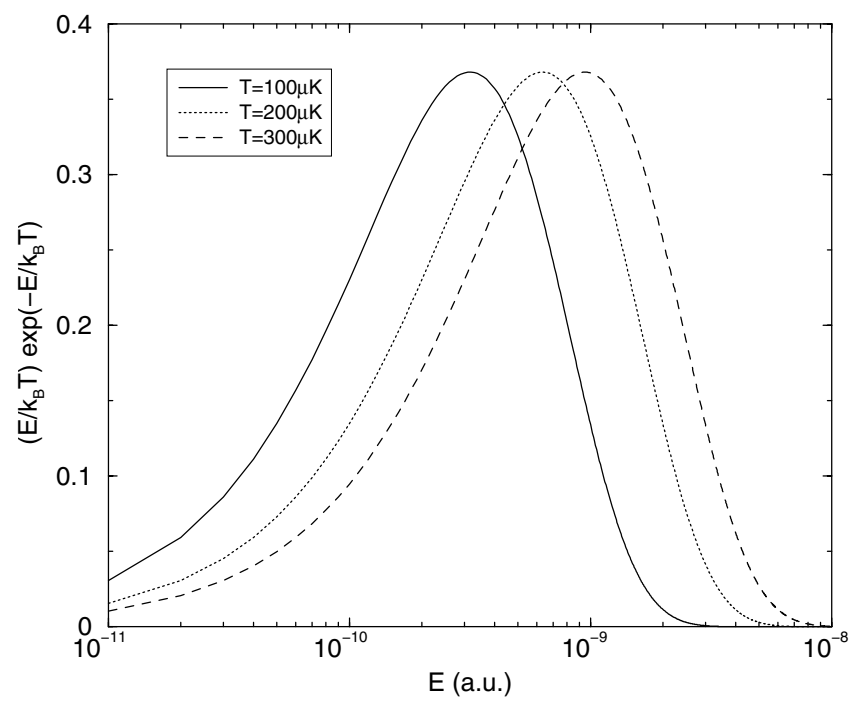

FIG. 6. Energy dependence of the distribution function needed to compute the trap loss rate coefficient for temperatures near those of the experiment [3].

mal rate coefficient for temperatures near those of the experiment. The $T=100 \mu \mathrm{K}$ curve samples very little of the energy region above $10^{-9}$ a.u. where deviation from Wigner threshold behavior begins to occur. The $T=200 \mu \mathrm{K}$ and $T$ $=300 \mu \mathrm{K}$ curves sample more of the above-threshold region; however, both curves are negligible in the region of the shape resonance. Therefore, if the decrease in the rate coefficient for $T>100 \mu \mathrm{K}$ is real, then the total quenching cross section would need to be considerably smaller than any of those shown in Fig. 4 for energies in the $10^{-9}-10^{-8}$ a.u. range. It is possible that hyperfine and magnetic field couplings could have an influence in this region and be contributing to the disagreement with experiment. It should be noted, however, that this discrepancy is limited to only two data points and suggests the need for additional experimental data.

\section{CONCLUSION}

We have implemented two significant improvements over our previous calculations [1,2] of collisional cross sections for spin-polarized $\mathrm{H}(2 s)$ atoms. Specifically, we have replaced the coupled states approximation with a coupledchannels formulation that properly handles nonadiabatic angular coupling at large distances. We have also included nonadiabatic radial coupling that arises from electrostatic dipole-quadrupole interactions between $(2 s, 2 p)$ and $(2 p, 2 p)$ states at long range. Calculated cross sections were found to be sensitive to both of these improvements.

The nonadiabatic coupling may be described in terms of a vector potential whose angular components do not vanish in the separated atom limit [6]. The molecular to atomic gauge transformation allows the angular components of the vector potential to fall off properly with distance, yielding nonadiabatic couplings that are well behaved in the asymptotic region. Inelastic effects induced by the vector potential at short distances are assumed to be small and are neglected. Nonadiabatic radial coupling is included through the $R$ dependence of the unitary transformation matrix (17). A set of calculations corresponding to 11 different values of $R_{0}$ was performed in order to study the influence of the nonadiabaticity on the cross sections as a function of distance. The results were found to be qualitatively similar with vanishing sensitivity at high energies. A trend toward smaller cross sections was found for $R_{0}<100$ a.u., which suggests the nonadiabatic coupling may be at least partly responsible for the disagreement with experiment. However, the calculated rate coefficients continue to be several times larger than the experimental error bars with little temperature variation in the region where the experiment suggests that there may be a significant decrease. Preliminary calculations that include hyperfine coupling but neglect nonadiabatic radial and angular coupling have produced only small changes to the cross sections. However, the cross sections may turn out to be significantly modified when nonadiabatic coupling is included together with the hyperfine interaction. This calculation requires an enlarged basis set that includes proton spin and is a natural extension of the formulation described here.

\section{ACKNOWLEDGMENTS}

The research of R.C.F. was supported by the National Science Foundation through Grant No. PHY-0554794 and a grant to ITAMP at Harvard University and Smithsonian Astrophysical Observatory. A.D. was supported by the Chemical Sciences, Geosciences and Biosciences Division of the Office of Basic Energy Sciences, Office of Science, U.S. Department of Energy. A.S. and Y.V. acknowledge financial support by the Deutsche Forschungsgemeinschaft DFG-Sa 936/1 within the SPP 1116. A.S. is grateful to the Stifterverband für die Deutsche Wissenschaft Programme Forschungsdozenturen and the Fonds der Chemischen Industrie for financial support. P.F. acknowledges support from the Swedish Research Council and from ITAMP.
[1] R. C. Forrey, R. Cote, A. Dalgarno, S. Jonsell, A. Saenz, and P. Froelich, Phys. Rev. Lett. 85, 4245 (2000).

[2] R. C. Forrey, S. Jonsell, A. Saenz, P. Froelich, and A. Dalgarno, Phys. Rev. A 67, 040701(R) (2003).

[3] D. Landhuis, L. Matos, S. C. Moss, J. K. Steinberger, K. Vant,
L. Willmann, T. J. Greytak, and D. Kleppner, Phys. Rev. A 67, 022718 (2003).

[4] Y. V. Vanne, A. Saenz, A. Dalgarno, R. C. Forrey, P. Froelich, and S. Jonsell, Phys. Rev. A 73, 062706 (2006).

[5] P. McGuire and D. J. Kouri, J. Chem. Phys. 60, 2488 (1974); 
P. McGuire, ibid. 62, 525 (1975); R. T. Pack, ibid. 60, 633 (1974).

[6] B. Zygelman, A. Dalgarno, and R. D. Sharma, Phys. Rev. A 49, 2587 (1994).

[7] T. G. Heil and A. Dalgarno, J. Phys. B 12, L557 (1979).
[8] S. Jonsell, A. Saenz, P. Froelich, R. C. Forrey, R. Cote, and A. Dalgarno, Phys. Rev. A 65, 042501 (2002).

[9] B. Zygelman, A. Dalgarno, and R. D. Sharma, Phys. Rev. A 50, 3920 (1994). 